DOI 10.1007/s00702-005-0351-z

J Neural Transm (2005) 112: 1647-1655

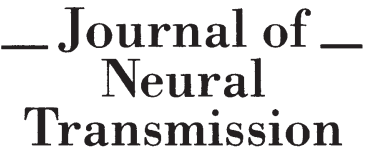

Printed in Austria

\title{
Functional imaging with positron emission tomography in multiple system atrophy
}

\author{
S. Gilman \\ Department of Neurology, University of Michigan, Ann Arbor, MI, USA \\ Received February 12, 2005; accepted June 18, 2005 \\ Published online August 5, 2005; (C) Springer-Verlag 2005
}

\begin{abstract}
Summary. Although the current guidelines for the clinical diagnosis of multiple system atrophy (MSA) do not require structural or functional brain imaging, investigations utilizing positron emission tomography (PET) have been helpful diagnostically in differentiating between MSA and primary autonomic failure; idiopathic Parkinson's disease; and sporadic olivopontocerebellar atrophy. These investigations have demonstrated different patterns of cerebral glucose utilization and of nigrostriatal projection abnormalities among these disorders and between the cerebellar and parkinsonian forms of MSA. Most of the studies have focused upon patients with well-established disease and none have examined the utility of PET imaging in early stage patients with follow-up of clinical course and autopsy verification to ensure accuracy of diagnosis and to determine the sensitivity and specificity of PET techniques for diagnosis. Recent PET studies have revealed denervation of myocardial post-ganglionic sympathetic neurons in some MSA patients, indicating that this disorder can affect the peripheral autonomic as well as the central nervous system. Investigations utilizing ligands to quantify central nervous system dopaminergic and cholinergic terminals have begun to provide insight into the neurochemical disorders that may underlie two of the sleep disturbances common in MSA, rapid eye movement sleep behavior disorder and obstructive sleep apnea.
\end{abstract}

Keywords: Positron emission tomography, functional imaging, multiple system atrophy.

\section{Introduction}

A consensus conference established the current diagnostic criteria for possible, probable, and definite multiple system atrophy (MSA) (Gilman et al., 1999b). The criteria are purely clinical, requiring neither structural nor functional imaging for diagnosis; nevertheless, imaging has proved useful as a research tool in this disorder. Positron emission tomography (PET) has contributed to clinical diagnosis by differentiating MSA from other related disorders, including 
Table 1. Radiotracer ligands used in PET investigations of MSA described in this review, their anatomical localizations, and the functions they can be used to measure

\begin{tabular}{|c|c|c|}
\hline Radiotracer ligand & Localization & Function measured \\
\hline$\left[{ }^{18} \mathrm{~F}\right]$ fluorodeoxyglucose & $\begin{array}{l}\text { Cerebral cortex, cerebellum, } \\
\text { thalamus, basal ganglia }\end{array}$ & $\begin{array}{l}\text { Local cerebral metabolic rates } \\
\text { for glucose }\end{array}$ \\
\hline$\left[{ }^{18} \mathrm{~F}\right]$ fluorodopa & $\begin{array}{l}\text { Striatal dopaminergic } \\
\text { presynaptic terminals }\end{array}$ & $\begin{array}{l}\text { Dopamine storage and dopa } \\
\text { decarboxylase activity }\end{array}$ \\
\hline$\left[{ }^{11} \mathrm{C}\right]$ dihydrotetrabenazine & $\begin{array}{l}\text { Striatal dopaminergic } \\
\text { presynaptic terminals }\end{array}$ & $\begin{array}{l}\text { Type } 2 \text { vesicular monoamine } \\
\text { transporter density }\end{array}$ \\
\hline$\left[{ }^{11} \mathrm{C}\right]$ diprenorphine & $\begin{array}{l}\text { Striatum, thalamus, amygdala, } \\
\text { cingulate gyrus, insular cortex, } \\
\text { frontal cortex }\end{array}$ & $\begin{array}{l}\text { Nonselective opoid receptor } \\
\text { sites (including } \mu, \kappa \text {, and } \delta \text { ) }\end{array}$ \\
\hline$\left[{ }^{11} \mathrm{C}\right]$ hydroxyephedrine & Heart & $\begin{array}{l}\text { Myocardial postganglionic } \\
\text { sympathetic terminal density }\end{array}$ \\
\hline $\begin{array}{l}\mathrm{N}-\left[{ }^{11} \mathrm{C}\right] \text { Methylpiperidin-4-yl } \\
\left.\left.\text { Propionate ([ }{ }^{11} \mathrm{C}\right] \mathrm{PMP}\right)\end{array}$ & $\begin{array}{l}\text { Cerebral cortex, thalamus, } \\
\text { striatum, pons }\end{array}$ & Utilization of acetylcholinesterase \\
\hline
\end{tabular}

pure autonomic failure (PAF), idiopathic Parkinson's disease (IPD), progressive supranuclear palsy (PSP) and sporadic olivopontocerebellar atrophy (sOPCA). Most of these investigations have involved subjects with well-characterized disease and not subjects in the earliest stages of the disease. Hence there are no published studies that have systematically examined the utility of PET imaging in early stage patients with determination of the subsequent clinical course and autopsy verification to ensure accuracy of diagnosis. Studies such as this would help evaluate the sensitivity and specificity of PET techniques for diagnosis. PET also has been used to characterize subgroups of MSA, detect involvement of other organ systems, and examine the pathogenesis of the sleep disturbances commonly found in this disease, rapid eye movement (REM) sleep behavior disorder (RBD) and obstructive sleep apnea (OSA). This review of functional imaging studies with PET in MSA briefly summarizes the results presented in representative research papers in these areas; this is not intended to be an exhaustive review of all PET imaging studies in MSA. Although this paper specifically concerns PET, other scanning techniques are mentioned briefly when necessary for clarity and accuracy in discussing the results of individual investigations. The other scanning techniques include computed tomography (CT), magnetic resonance imaging (MRI) and single photon emission computed tomography (SPECT). Table 1 provides a brief summary of the radiotracer ligands used in PET investigations of MSA discussed in this paper.

\section{Results and discussion}

\section{Differential diagnosis of MSA and PAF}

Fulham et al. (1991) used computed tomography (CT), magnetic resonance imaging (MRI) and PET with $\left[{ }^{18} \mathrm{~F}\right]$ fluorodeoxyglucose (FDG-PET) to characterize the neuroimaging features of MSA and PAF. They also examined the utility of these techniques in distinguishing between these disorders. They investigated 30 patients with MSA and 15 with PAF. The MSA group included eight patients 
with MSA of the cerebellar type (MSA-C), seven patients with MSA of the parkinsonian type (MSA-P) and 15 patients who had both cerebellar and parkinsonian features. In the MSA but not the PAF patients they found cerebellar atrophy on CT and MRI, signal hypointensity in the posterolateral putamen on MRI, and a generalized reduction in local cerebral metabolic rates for glucose (1CMRglc) with FDG-PET. Glucose hypometabolism was most prominent in the cerebellum, brainstem, striatum, and frontal and motor regions of the cerebral cortex. They concluded that neuroimaging studies clearly distinguish MSA from PAF.

\section{Distinguishing between MSA, PD and PSP}

Burn et al. (1994) utilized $\left[{ }^{18} \mathrm{~F}\right]$ fluorodopa with PET (FD-PET), a means of quantifying the density of presynaptic dopaminergic terminals, to examine the pattern of nigrostriatal dopaminergic dysfunction in patients with parkinsonian syndromes. The group included 27 normal control subjects, 28 patients with IPD, 25 with MSA, and 10 with PSP (termed Steele-Richardson-Olszewski syndrome in this paper). The authors used a discriminant function analysis in assessing the ability of FD-PET to categorize individual parkinsonian patients based upon tracer uptake in the caudate nucleus and putamen. The discriminant function analysis assigned all control subjects to a normal category. One patient with a parkinsonian syndrome out of the 63 studied was classified as normal on the basis of PET findings, although this patient's scan showed significantly reduced uptake in the putamen. Discriminant function analysis was less effective than clinical criteria at distinguishing different categories of parkinsonian syndrome. Patients labeled clinically as having typical or atypical parkinsonian syndromes were assigned the same category on PET criteria $64 \%$ and $69 \%$ of the time, respectively. When the three categories of IPD, MSA, and PSP were considered together, clinical and FD-PET findings correlated in $64 \%$ of patients assigned a diagnosis of IPD and $70 \%$ of those given a diagnosis of PSP. MSA was less readily discriminated; these patients were assigned to MSA, IPD, and PSP groups with equal frequency. Hence even in well-established patients, FD-PET did not differentiate sharply between these three disorders, at least in comparison with clinical categorization.

Otsuka et al. (1997) reported PET examinations of striatal FD-PET uptake and FDG-PET in nine patients with MSA and 15 with IPD as compared with equivalent numbers of normal controls. Five of the MSA patients had OPCA, two had striatonigral degeneration and two had MSA-P (described as Shy-Drager syndrome). FD uptake ratios to the occipital cortex in MSA at $120 \mathrm{~min}$ after administration were $2.07 \pm 0.31$ (mean \pm SD) in the caudate nucleus $(\mathrm{CN})$ and $1.96 \pm 0.29$ in the putamen (PUT), which were decreased compared to controls $(2.72 \pm 0.11 \mathrm{CN}, 2.71 \pm 0.10 \mathrm{PUT})$. In the IPD patients the values were $2.07 \pm 0.36 \mathrm{CN}$ and $1.74 \pm 0.24$ PUT, which was also decreased, but the decrease in the putamen was greater in IPD than MSA. A caudate-putamen index (CPI) was developed based upon differences in the uptakes in the CN and PUT divided by the CN. The CPI was $5.6 \pm 4.6$ in the MSA patients and $14.8 \pm 5.4$ in the IPD patients. LCMRglc in IPD showed no difference from the normal 
controls. In MSA, 1CMRglc was decreased in the frontal and the temporal cortical regions and in the $\mathrm{CN}$, PUT, cerebellum and brainstem as compared to controls. There was considerable overlap in lCMRglc between MSA and normal control groups, however. The authors concluded that FD-PET is useful for distinguishing MSA from IPD, and appears more useful than FDG-PET.

\section{Comparisons of OPCA and MSA-C}

Gilman et al. (1994) used FDG-PET to examine lCMRglc in patients with MSA, sOPCA, and dominantly inherited olivopontocerebellar atrophy (dOPCA) in comparison with normal control subjects. In MSA, lCMRglc was significantly decreased in the brainstem, cerebellum, putamen, thalamus, and cerebral cortex as compared to the normal controls. In sOPCA, 1CMRglc was significantly decreased in the brainstem, cerebellum, putamen, thalamus, and cerebral cortex. In dOPCA, lCMRglc was significantly decreased in the brainstem and cerebellum but not in the other structures. Examination of 1CMRglc normalized to the cerebral cortex in comparison with normal controls revealed in MSA significant decreases in the brainstem, cerebellum, and putamen, but in both sOPCA and dOPCA, significant decreases were found only in the brainstem and cerebellum. The authors concluded that these three disorders all show a marked decrease of lCMRglc in the brainstem and cerebellum but differ in the degree of hypometabolism in forebrain and cerebral cortex. The results were consistent with the possibility that, in many cases, sOPCA will evolve into MSA.

In a later investigation, Gilman et al. (1996) used $( \pm)-\left[{ }^{11} \mathrm{C}\right]$ dihydrotetrabenazine, a newly developed ligand for the type 2 vesicular monoamine transporter (VMAT2), with PET (DTBZ-PET) to examine striatal monoaminergic presynaptic terminals in four MSA and eight sOPCA patients compared with nine normal control subjects. Specific binding in the striatum was significantly reduced in MSA as compared with the normal controls, with average reductions of $61 \%$ in the $\mathrm{CN}(\mathrm{p}=0.002)$ and $58 \%$ in the PUT $(\mathrm{p}=0.009)$. Smaller reductions were found in the sOPCA group, averaging $26 \%$ in the $\mathrm{CN}(\mathrm{p}=0.05)$ and $24 \%$ in the PUT $(p=0.11)$. Mean blood-to-brain DTBZ transport $\left(\mathrm{K}_{1}\right)$ was significantly different between groups only in the cerebellum, with values for the sOPCA group diminished compared with the normal control group. Cerebellar $\mathrm{K}_{1}$ was not significantly decreased in the MSA group. The finding of reduced striatal VMAT2 in sOPCA suggested nigrostriatal pathology, indicating that some may later develop symptomatic extrapyramidal disease and consequently progress to MSA.

Subsequently, Gilman et al. (1999a) examined the density of striatal presynaptic monoaminergic terminals using the $(+)$ enantiomer for the VMAT2, (+)-[ ${ }^{11}$ C]DTBZ, with PET in eight MSA-P, eight MSA-C, six sOPCA patients and seven normal controls. As compared with controls, binding was significantly reduced in the PUT of all patient groups, and binding values were significantly lower in MSA-P than in MSA-C, and significantly lower in MSA-C than in SOPCA. Mean $\mathrm{K}_{1}$ was significantly decreased in the PUT of all patient groups and in the cerebellar hemispheres of MSA-C and sOPCA but not MSA-P groups, compared with controls. Significant negative correlations were found between striatal binding and the severity of parkinsonian features and 
between cerebellar $\mathrm{K}_{1}$ and the severity of cerebellar dysfunction. The results indicated differences between MSA-P and MSA-C groups reflecting differential severity in degeneration of nigrostriatal and cerebellar systems. The findings also showed that some sOPCA patients have subclinical nigrostriatal dysfunction and are at risk of developing MSA with disease progression.

Rinne et al. (1995) examined 10 patients with sOPCA and autonomic failure in comparison to normal control subjects using PET with $\left[{ }^{11} \mathrm{C}\right]$ diprenorphine (a nonspecific opioid antagonist) and $\left[{ }^{18} \mathrm{~F}\right] \mathrm{FD}$. The mean caudate-occipital uptake ratio for $\left[{ }^{11} \mathrm{C}\right.$ ]diprenorphine was significantly reduced to $88 \%$ and the putamenoccipital uptake ratio to $85 \%$ of the control values. Individually, four of the 10 patients had significantly reduced opioid binding in the PUT. Mean PUT FD-PET uptake was significantly diminished (to $71 \%$ of the control mean); individually seven patients had significantly reduced uptake. The authors found a significant positive correlation between putamen-occipital uptake ratios for $\left[{ }^{11} \mathrm{C}\right]$ diprenorphine and PUT uptake of FD. They concluded that subclinical nigrostriatal dysfunction occurs in a majority of patients with sOPCA, in accordance with its status as part of the spectrum of MSA. The difficulty with this conclusion is that their patients all had autonomic failure, indicating that they already had MSA of the cerebellar type. Hence their results did not demonstrate that sOPCA is necessarily part of the spectrum of MSA.

\section{Cardiac imaging of $P D$ and $M S A$}

Metaiodobenzylguanidine (MIBG) is a norepinephrine analogue that is taken up and stored in sympathetic nerve endings, and when labeled with [ $\left.{ }^{123} \mathrm{I}\right]$, MIBG can be used with single photon emission computed tomography (SPECT) to examine peripheral postganglionic sympathetic neuronal terminals. Multiple imaging studies with [ ${ }^{123}$ I]MIBG in IPD patients have shown decreased cardiac uptake, indicating myocardial postganglionic sympathetic dysfunction (Takatsu et al., 2000; Reinhardt et al., 2000; Druschky et al., 2000; Braune et al., 1999; Orimo et al., 1999; Satoh et al., 1999; Yoshita, 1998). Most investigations have involved IPD patients in Hoehn and Yahr stages 3-5, but some have shown denervation at earlier stages, including patients with and without autonomic dysfunction (Courbon et al., 2003). At Hoehn and Yahr stage 1, however, uptake has been reported to be normal (Satoh et al., 1999). In several MIBG investigations of MSA, myocardial uptake was no different from normal controls (Taki et al., 2000; Braune et al., 1999; Takatsu et al., 2000; Reinhardt et al., 2000). In a few reports, however, MSA subjects showed reduced uptake as compared to normal controls, although IPD patients in the same studies showed much greater levels of denervation (Yoshita, 1998; Druschky et al., 2000; Hirayama et al., 1995).

Although investigations with SPECT and MIBG have provided important information about peripheral autonomic involvement in IPD, the tracer has several drawbacks. Single-photon scintigraphic imaging devices have inherent shortcomings in spatial resolution and measurement of tissue radioactivity that limit attempts to quantify radiotracer ligand uptake. Most investigations utilize the ratio of $\left[{ }^{123}\right.$ I]MIBG activity in the heart divided by activity in the mediastinum to measure cardiac nerve density. In contrast, PET imaging offers excel- 
lent spatial resolution and accurate measurements of tissue radioactivity concentrations. PET imaging with the sympathetic nerve tracer $\left[{ }^{11} \mathrm{C}\right]$ meta-hydroxyephedrine $\left(\left[{ }^{11} \mathrm{C}\right] \mathrm{HED}\right)$ not only permits precise quantification of cardiac sympathetic nerve density, but also allows for detailed assessment of any regional variation in left ventricular cardiac sympathetic innervation (Raffel and Wieland, 2001). For these reasons, PET studies have been initiated with $\left[{ }^{11} \mathrm{C}\right]$ hydroxyephedrine (HED), which enters myocardial sympathetic endings primarily through specific uptake into the neuronal norepinephrine transporter. Berding et al. (2003) reported the results of a pilot study investigating seven patients, two with MSA and orthostatic hypotension $(\mathrm{OH})$, three with probable IPD and $\mathrm{OH}$, one with probable IPD without $\mathrm{OH}$, and one with possible IPD without $\mathrm{OH}$. Ratios of HED uptake in the myocardium to the liver at 5 and 40 minutes post injection were calculated. Also, tracer influx rates $\left(\mathrm{K}_{1}\right)$ were computed based upon kinetic modeling. Compared with MSA patients, IPD patients with $\mathrm{OH}$ showed lower uptake ratios at both time points and lower $\mathrm{K}_{1}$ values. The patient with probable IPD without $\mathrm{OH}$ showed intermediate values of both uptake ratios and $\mathrm{K}_{1}$. In the patient with possible IPD, uptake ratios and $\mathrm{K}_{1}$ values did not differ from those observed in MSA. The authors concluded that measurement of myocardial innervation may contribute to the differential diagnosis of parkinsonian disorders and suggested a need for quantitative pathophysiological imaging, particularly at early stages of disease.

Gilman et al. (2004) recently reported preliminary results of an investigation utilizing HED for cardiac imaging of five patients with MSA, two with progressive supranuclear palsy (PSP) and 10 normal subjects using HED with PET. The HED dose of $20 \pm 1 \mathrm{mCi}$ was injected intravenously and a dynamic sequence of scans was acquired for 40 minutes. Cardiac images were sliced by computer algorithm into eight short-axis images, each subdivided into 60 angular sectors. Tissue concentrations of HED at 30 to 40 minutes post-injection were normalized by dividing by the integral of the blood time-activity curve, providing a retention index (RI) for each myocardial region, reflecting the ability of the sympathetic neurons to take up and store norepinephrine. Two MSA patients had RI values over 2.5 standard deviations below normal levels for all 480 sectors. One of these expired months after the study, and brain autopsy verified the diagnosis. In the third MSA patient, $20 \%$ of the sectors showed RI values over 2.5 SD below normal levels. The two remaining MSA patients and both PSP patients had RI values within normal levels throughout. The findings show that some MSA patients have severe post-ganglionic sympathetic denervation of the heart whereas PSP patients have normal innervation. This indicates that peripheral sympathetic neurons can be affected in MSA.

\section{Pathophysiology of sleep disorders in MSA}

MSA is associated with multiple sleep disorders, among them REM sleep behavior disorder (RBD) and obstructive sleep apnea (OSA). In two recent exploratory investigations, Gilman et al. (2003a, b) examined the neurochemical basis of these disturbances. Thirteen MSA patients were studied and compared with normal control subjects. The first study (Gilman et al., 
2003a) examined the neurochemical abnormalities that may underlie RBD. Nocturnal, laboratory-based polysomnography (PSG) was used to rate the severity of REM atonia loss by the percentage of REM sleep with tonically increased electromyographic (EMG) activity and the percentage of REM sleep with phasic EMG bursts. PET with $(+)-\left[{ }^{11} \mathrm{C}\right] \mathrm{DTBZ}$ was used to determine the density of striatal presynaptic monoaminergic terminals, and SPECT with $(-)-5-\left[{ }^{123} I\right]$ iodobenzovesamicol $\left(\left[{ }^{123} \mathrm{I}\right] \mathrm{IBVM}\right)$ to measure the density of thalamic presynaptic cholinergic terminals. The MSA subjects showed decreased mean DTBZ binding in the striatum $(\mathrm{p}<0.0001)$ compared with 15 normal control subjects and decreased IBVM binding in the thalamus $(p<0.001)$ compared with 12 normal control subjects. Moreover, in the MSA group, striatal DTBZ binding was inversely correlated with the severity of REM atonia loss $(\mathrm{p}=0.003)$. Thalamic IBVM binding, however, was not correlated to the severity of REM atonia loss. The study suggested that decreased nigrostriatal dopaminergic projections may contribute to RBD in MSA.

The second study (Gilman et al., 2003b) explored the neurochemical basis of OSA utilizing the same patients, normal control subjects, and PET/SPECT ligands. The investigators used the same nocturnal, laboratory-based PSG, but in this study they computed the apnea-hypopnea index during sleep to rate the severity of OSA. In the MSA group, thalamic IBVM binding was inversely correlated with the severity of OSA $(p=0.011)$. Striatal DTBZ binding was not correlated with the severity of OSA $(p=0.19)$. These findings suggested that decreased pontine cholinergic projections may contribute to OSA in MSA.

These studies were designed with the hypothesis that denervation of both dopaminergic nigrostriatal terminals and pontine cholinergic nuclei would show a relationship to RBD severity. Multiple investigations in experimental animals indicate the important function of these nuclei in REM sleep and are likely to be important in RBD (Rye, 1997). At the time of publication of the studies cited above, we had no means of imaging these brainstem nuclei, as IBVM with SPECT provided inadequate resolution to analyze brainstem cholinergic content. In a current ongoing investigation, however, we have utilized the cholinergic marker N- $\left[{ }^{11} \mathrm{C}\right]$ Methylpiperidin-4-yl Propionate $\left(\left[{ }^{11} \mathrm{C}\right] \mathrm{PMP}\right)$, and preliminary data suggest participation of these cholinergic neurons in RBD of MSA patients. These findings have not as yet been published.

\section{Future studies of MSA with PET}

The studies reviewed above have shown that PET provides valuable information about cerebral neurochemical changes in patients with MSA. Information from these studies can be used to understand the pathophysiological basis of some of the neurological disorders encountered clinically and perhaps can be useful in differential diagnosis of the various parkinsonian syndromes. PET scanning is a specialized technique that requires expensive equipment and highly trained personnel but can produce multiple types of tracers designed for specific targets. Although more limited in tracer availability and resolution, SPECT scanning requires fewer specialized personnel and is less expensive and more widely accessible. Future innovative studies of MSA are likely to come 
from PET centers utilizing unique specialized tracers, but important, useful information will come from SPECT studies as well. Some of the future studies perhaps will utilize functional imaging in the early stages of the common parkinsonian syndromes to determine the sensitivity and specificity of imaging in predicting the outcome of these disorders with verification by both clinical course and autopsy study.

\section{References}

Berding G, Schrader CH, Peschel T, van den Hoff J, Kolbe H, Meyer GJ, Dengler R, Knapp WH (2003) [N-methyl ${ }^{11}$ C]meta-Hydroxyephedrine positron emission tomography in Parkinson's disease and multiple system atrophy. Eur J Nucl Med 30: 127-131

Braune S, Reinhardt M, Schnitzer R, Riedel A, Lücking CH (1999) Cardiac uptake of [ $\left.{ }^{123} \mathrm{I}\right] \mathrm{MIBG}$ separates Parkinson's disease from multiple system atrophy. Neurology 53: 1020-1025

Burn DJ, Sawle GV, Brooks DJ (1994) Differential diagnosis of Parkinson's disease, multiple system atrophy, and Steele-Richardson-Olszewski syndrome: discriminant analysis of striatal ${ }^{18}$ F-dopa PET data. J Neurol Neurosurg Psychiatry 57: 278-284

Courbon F, Brefel-Courbon C, Thalamas C, Alibelli MJ, Berry I, Montastruc JL, Rascol O, Senard JM (2003) Cardiac MIBG scintigraphy is a sensitive tool for detecting cardiac sympathetic denervation in Parkinson's disease. Mov Disord 18: 890-897

Druschky A, Hilz MJ, Platsch G, Radespiel-Tröger M, Druschky K, Kuwert T, Neundörfer B (2000) Differentiation of Parkinson's disease and multiple system atrophy in early disease stages by means of I-123-MIBG-SPECT. J Neurol Sci 175: 3-12

Fulham MJ, Dubinsky RM, Polinsky RJ, Brooks RA, Brown RT, Curras MT, Baser S, Hallett M, Di Chiro G (1991) Computed tomography, magnetic resonance imaging and positron emission tomography with $\left[{ }^{18} \mathrm{~F}\right]$ fluorodeoxyglucose in multiple system atrophy and pure autonomic failure. Clin Auton Res 1: 27-36

Gilman S, Frey KA, Koeppe RA, Junck L, Little R, Vander Borght TM, Lohman M, Martorello S, Lee LC, Jewett DM, Kilbourn MR (1996) Decreased striatal monoaminergic terminals in olivopontocerebellar atrophy and multiple system atrophy demonstrated with positron emission tomography. Ann Neurol 40: 885-892

Gilman S, Koeppe RA, Chervin R, Consens F, Little R, An H, Junck L, Heumann M (2003a) REM sleep behavior disorder is related to striatal monoaminergic deficit in MSA. Neurology 61: $29-34$

Gilman S, Koeppe RA, Chervin R, Consens F, Little R, An H, Junck L, Heumann M (2003b) Obstructive sleep apnea is related to a thalamic cholinergic deficit in MSA. Neurology 61: 35-39

Gilman S, Koeppe RA, Junck L, Kluin KJ, Lohman M, St Laurent RT (1994) Patterns of cerebral glucose metabolism detected with PET differ in multiple system atrophy and olivopontocerebellar atrophy. Ann Neurol 36: 166-175

Gilman S, Koeppe RA, Junck L, Little R, Kluin KJ, Heumann M, Martorello S, Johanns J (1999a) Decreased striatal monoaminergic terminals in multiple system atrophy detected with PET. Ann Neurol 45: 769-777

Gilman S, Low PA, Quinn N, Albanese A, Ben-Shlomo Y, Fowler CJ, Kaufmann H, Klockgether T, Lang AE, Lantos PL, Litvan I, Mathias CJ, Oliver E, Robertson D, Schatz I, Wenning GK (1999b) Consensus statement on the diagnosis of multiple system atrophy. J Neurol Sci 163: 94-98

Gilman S, Raffel DA, Koeppe RA, Little R, An H, Junck L, Heumann M (2004) Cardiac sympathetic innervation in multiple system atrophy and progressive supranuclear palsy. Mov Disord 19: 1118 (Abstract)

Hirayama M, Hakusui S, Koike Y, Ito K, Kato T, Ikeda M, Hasegawa Y, Takahashi A (1995) A scintigraphical qualitative analysis of peripheral vascular sympathetic function with meta- $\left[{ }^{123}\right.$ I]iodobenzylguanidine in neurological patients with autonomic failure. J Auton Nerv Syst 53: 230-234 
Langer O, Halldin C (2002) PET and SPET tracers for mapping the cardiac nervous system. Eur J Nucl Med 29: 416-434

Orimo S, Ozawa E, Nakade S, Sugimoto T, Mizusawa H (1999) (123)I-metaiodobenzylguanidine myocardial scintigraphy in Parkinson's disease. J Neurol Neurosurg Psychiatry 67: $189-194$

Otsuka M, Kuwabara Y, Ichiya Y, Hosokawa S, Sasaki M, Yoshida T, Fukumura T, Kato M, Masuda K (1997) Differentiating between multiple system atrophy and Parkinson's disease by positron emission tomography with ${ }^{18}$ F-dopa and ${ }^{18}$ F-FDG. Ann Nucl Med 11: 251-257

Raffel DM, Wieland DM (2001) Assessment of cardiac sympathetic nerve integrity with positron emission tomography. Nucl Med Biol 28: 541-559

Reinhardt MJ, Jüngling FD, Krause TM, Braune S (2000) Scintigraphy differentiation between two forms of primary dysautonomia early after onset of autonomic dysfunction: value of cardiac and pulmonary iodine-123 MIBG uptake. Eur J Nucl Med 27: 595-600

Rinne JO, Burn DJ, Mathias CJ, Quinn NP, Marsden CD, Brooks DJ (1995) Positron emission tomography studies on the dopaminergic system and striatal opioid binding in the olivopontocerebellar atrophy variant of multiple system atrophy. Ann Neurol 37: 568-573

Rye DB (1997) Contributions of the pedunculopontine region to normal and altered REM sleep. Sleep 20: 757-788

Satoh A, Serita T, Seto M, Tomita I, Satoh H, Iwanaga K, Takashima H, Tsujihata M (1999) Loss of ${ }^{123}$ I-MIBG uptake by the heart in Parkinson's disease: assessment of cardiac sympathetic denervation and diagnostic value. J Nucl Med 40: 371-375

Takatsu H, Nagashima K, Murase M, Fujiwara H, Nishida H, Matsuo H, Watanabe S, Satomi K (2000) Differentiating Parkinson's disease from multiple-system atrophy by measuring cardiac iodine-123 metaiodobenzylguanidine accumulation. JAMA 284: 44-45

Taki J, Nakajima K, Hwang EH, Matsunari I, Komai K, Yoshita M, Sakajiri K, Tonami N (2000) Peripheral sympathetic dysfunction in patients with Parkinson's disease without autonomic failure is heart selective and disease specific. Eur J Nucl Med 27: 566-573

Yoshita M (1998) Differentiation of idiopathic Parkinson's disease from striatonigral degeneration and progressive supranuclear palsy using iodine-123 meta-iodobenzylguanidine myocardia scintigraphy. J Neurol Sci 155: 60-67

Author's address: S. Gilman, M.D., F.R.C.P., Department of Neurology, University of Michigan, 300 North Ingalls St. 3D15, Ann Arbor, Michigan 48109-0489, USA, e-mail: sgilman@umich.edu 